\title{
Experimental Verification for the Application Feasibility of Buckingham Pi Theorem in Designing a Scaled Model of Flagellated Swimmer with Traditional Resistive Force Theory
}

\author{
Ouliang Jin \\ Huazhong University of Science and Technology, Wuhan, China \\ Email: u201714356@hust.edu.cn
}

How to cite this paper: Jin, O.L. (2021) Experimental Verification for the Application Feasibility of Buckingham Pi Theorem in Designing a Scaled Model of Flagellated Swimmer with Traditional Resistive Force Theory. Modern Mechanical Engineering, 11, 83-72.

https://doi.org/10.4236/mme.2021.113004

Received: February 9, 2021

Accepted: August 28, 2021

Published: August 31, 2021

Copyright $\odot 2021$ by author(s) and Scientific Research Publishing Inc. This work is licensed under the Creative Commons Attribution International License (CC BY 4.0).

http://creativecommons.org/licenses/by/4.0/

\begin{abstract}
The microstructure of bacterial is an important reference to design underwater swimmers. Applying Buckingham Pi Theorem is a possible method to build a scaled model based on a theoretical flagellum designed with traditional Resistive Force Theory. By making a scaled model, the forward velocity could be obtained to verify the application feasibility of Buckingham Pi Theorem in such designs. The optimal value of the model's pitch angle can be calculated with the traditional RFT as well as the forward velocity. Comparing the experimental results with the results of theoretical calculations, it is found that the experimental and calculated results are consistent, which means Buckingham Pi Theorem works well in such design of underwater swimmer.
\end{abstract}

\section{Keywords}

Micro AUV, Bionic-Robot, Flagellum Motor, Low Reynolds Number

\section{Introduction}

In recent years, micro underwater machines in low Reynolds number environment are widely studied and applied in different fields such as medical treatment and drug delivery [1] [2] [3]. Many factors should be taken into consideration in order to design a good swimmer at low Reynolds number environment. For example, a reciprocal motion should be avoided because scallop theory [4] 
proves that reciprocal-movement swimmers will stay where it is after it finishes a motion cycle.

Thus, designing a brand-new swimming structure working at a low Re number environment may cost much time and effort. An effective way to simplify the design process is to draw lessons from real lives. Bacterial flagellum, a structural model abstracted from microbes represented by $E$. coli, is a typical example. In this paper, we will build a scaled model of flagellum motor and design its geometry parameters in the experiments.

Traditional Resistive Force Theory (RFT) was firstly studied and applied to the motion of flagellated bacteria by Holwill and Burge [5]. It is a classic theory used to analyze flagellum motors' dynamic and kinetic parameters. Based on the traditional RFT, we could build a mathematical model for a flagellum motor model and calculate its theoretical speed.

Some other work is necessary if we want to apply the model in a real application, because the size of the model always needs to be adjusted. When studying such problems about model scaling in fluidic environment, people tend to use Buckingham Pi Theorem [6].

However, we just use Buckingham Pi theorem based on deduction proof. Whether Buckingham Pi Theorem work as well in designing a scaled model of flagellated swimmer with traditional RFT? If its application feasibility can be proved, flagellum motor structure of more different sizes can be properly designed to solve more complexes real problems in daily life, industrial manufacture and other fields. Similarly, the verification way mentioned in this paper can also be referred to verify the application feasibility of Buckingham Pi Theorem in other structural designs, especially those bionic structures.

In this paper, we mainly try to verify application feasibility of Buckingham Pi Theorem in the scaled design of a flagellated model via an experiment with a theoretical model and a real scaled model. With a series of inferential proofs are proposed, creating a fluidic environment with an extreme low Reynolds number is not a necessity for this verification experiment.

\section{Result}

\subsection{Theoretical Calculation}

\subsubsection{Traditional RFT Calculation}

Figure 1 and Figure 2 show the approximate model structure and the basic load conditions. To calculate the speed of the scaled model, we should firstly build up an original mathematical model and derive a formula for calculating the velocity directly. Traditional RFT is a proper reference and Magariyama's work summarized its mathematical expression as follows [7].

\section{Equations of motions}

$$
F_{c}+F_{f}=0
$$

Force on cell body 


$$
F_{c}=\alpha_{c} v
$$

Drag force acting on the flagellum tail

$$
F_{f}=\alpha_{f} v+\gamma_{f} \omega_{f}
$$

Table 1 shows all symbols mentioned in above formulas and what parameters they represent.

We can solve for the velocity, $v$, from the Equations (1)-(3) as follows.

$$
v=-\frac{\omega_{f} \gamma_{f}}{\alpha_{c}+\alpha_{f}}
$$

In Equation (4), values of parameters including drag coefficients of cell body and flagellum tail remain unknown.

Happel and Brenner once solved drag coefficients of the cell body [8]. However, those formulas are applied to do such calculation for a spheroid cell body. In this paper, the cell body of the scaled body is a cylinder. As a result, we choose to take an approximated calculation. Dimensional parameters of a spheroid are replaced by corresponding dimensional parameters of a cylinder. Equation (5) is adapted from the formula mentioned in Magariyama's paper [7].

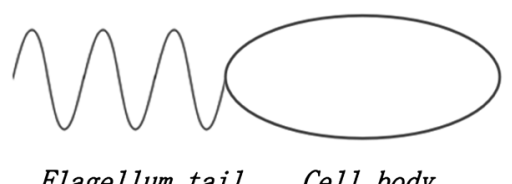

Flagellum tail Cell body

Figure 1. Schematic diagram of an E. coli cell.

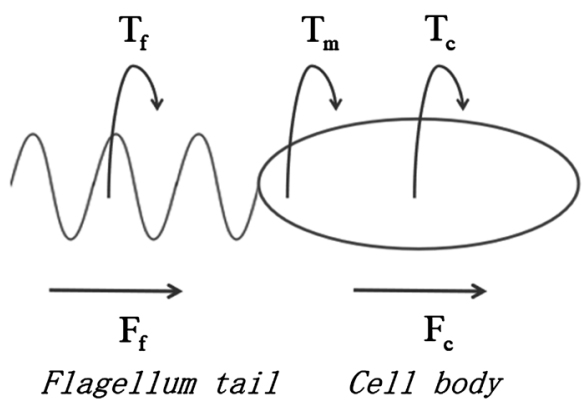

Figure 2. Force diagram exerted on a micro swimmer.

Table 1. Symbols mentioned in Equations (1)-(3) and what they represent.

\begin{tabular}{cc}
\hline Symbols & Parameters \\
\hline$F_{c}$ & Force on cell body \\
$F_{f}$ & Drag force acting on tail \\
$V$ & Swimming velocity of motor \\
$\alpha_{c}$ & Axial drag coefficient of cell body \\
$\alpha_{f}$ & Axial drag coefficient of tail \\
$\gamma_{f}$ & Rotation tangential drag coefficient of tail \\
$\omega_{f}$ & Rotating speed of tail \\
\hline
\end{tabular}




$$
\alpha_{c}=-6 \pi \mu r_{c}\left\{1-\frac{1}{5}\left(1-\frac{l_{c}}{r_{c}}\right)\right\}
$$

Table 2 shows all symbols mentioned in Equation (5), and what parameters they represent.

The flagellum tail of the scaled model is shaped like a helical thin filament. Holwill, Burge [5] and Magariyama [9] once studied about that. We could calculate the drag coefficients according to their work as follows.

$$
\begin{aligned}
& \alpha_{f}=\frac{2 \pi \mu L}{\left(\log \left(r_{f} / 2 p\right)+1 / 2\right)\left(4 \pi^{2} r^{2}+p^{2}\right)} \times\left(8 \pi^{2} r^{2}+p^{2}\right) \\
& \gamma_{f}=\frac{2 \pi \mu L}{\left(\log \left(r_{f} / 2 p\right)+1 / 2\right)\left(4 \pi^{2} r^{2}+p^{2}\right)} \times\left(8 \pi^{2} r^{2}+p^{2}\right)
\end{aligned}
$$

Table 3 shows all symbols mentioned in Equation (6)-(7), and what parameters they represent.

With Equations (4)-(7), we can calculate the speed of the theoretical model. All parameters and their values will be presented in 3.1 .

\subsubsection{Buckingham Pi Theorem Calculation [4]}

With traditional RFT, we are now able to calculate the swimming speed as above. After that, we can start to verify application feasibility of Buckingham Pi Theorem. Assuming that Buckingham Pi Theorem will work, we will build up a mathematical model for the scaling process.

During the process of scaling, we need firstly to list all variables. Our target is to calculate the forward speed ( $V$ ) of the scaled model which is designed based on the original mathematical model. Except the forward speed, the dimension of the model $(L)$, the rotating speed of the flagellum tail $\left(\omega_{f}\right)$ and the viscosity of the environment $(\mu)$ are also variables which should be taken into consideration. All variables and their units are listed in Table 4.

Table 2. Symbols mentioned in Equation (5) and what they represent.

\begin{tabular}{cc}
\hline Symbols & Parameters \\
\hline$r_{c}$ & Bottom radius of cell body \\
$I_{c}$ & Length of cell body \\
$\mu$ & Viscosity of environment liquid \\
\hline
\end{tabular}

Table 3. Symbols mentioned in Equation (6)-(7) and what they represent.

\begin{tabular}{cc}
\hline Symbols & Parameters \\
\hline$p$ & Helix pitch of tail \\
$r$ & Radius of helix \\
$r_{f}$ & Radius of the tail \\
$L$ & Length of tail \\
\hline
\end{tabular}


Table 4. All relative variables in speed calculation and their units.

\begin{tabular}{ccc}
\hline Variable & Unit & Dimension \\
\hline$V$ & $\mathrm{~m} \cdot \mathrm{s}^{-1}$ & $\mathrm{~L} \cdot \mathrm{T}^{-1}$ \\
$L$ & $\mathrm{~m}$ & $\mathrm{~L}$ \\
$\omega$ & $\mathrm{rad} \cdot \mathrm{s}^{-1}$ & $\mathrm{~T}^{-1}$ \\
$\mu$ & $\mathrm{Pa} \cdot \mathrm{s}$ & $\mathrm{M} \cdot \mathrm{L}^{-1} \cdot \mathrm{T}^{-1}$ \\
\hline
\end{tabular}

Here we achieve the functional relationship model as follow.

$$
V \sim f(L, \omega, \mu)
$$

With dimensional analysis and $\mathrm{Pi}$ terms solution [4], we can finally get the exact relationship between these parameters as follow.

$$
V \propto \omega \cdot L
$$

Therefore, we can calculate forward speed of the scaled model with the formula.

$$
V_{S}=\frac{\omega_{S} L_{S}}{\omega_{O} L_{O}} V_{O}
$$

All parameters with a $O$ subscript are in relation to the original model, while all parameters with a $S$ subscript are in relation to the original model.

\section{Theoretical Model Building}

\subsection{Parameters Determination}

A Matlab program with GUI controls is designed to calculate the speed of the mathematical model of a flagellum motor based on traditional RFT. The main code of the program will be presented in Appendix 1.

Figure 3 shows a Matlab GUI panal which includes all parameters and values of the original model and the calculation result of the speed.

Here, we need to check the Reynolds number to ensure that original model swims in the low Re number environment, because traditional RFT is only applicable to the calculation of flagellum models swimming in low Re number environment. This model is designed to swim in the water. Re number of the original model's swimming environment is calculated as Formula (9) shows.

$$
\operatorname{Re}_{o}=\frac{\rho v d}{\mu}=\frac{1000 \times 5.56 \times 10^{-5} \times 5 \times 10^{-6}}{1 \times 10^{-3}} \approx 3 \times 10^{-4}
$$

The Re number of $3 \times 10^{-4}$ can be absolutely viewed as a low Re number. That means traditional RFT can work in the designed original model's calculation.

Additionally, the whole length of the tail consists of two helix pitch. This is determined ahead since it will ease the manufacture of the helix tail in the latter steps. We determine all parameters' values shown in Figure 3 except the pitch angle. The value of pitch angle is calculated based on the length and the helix number of the flagellum tail. 


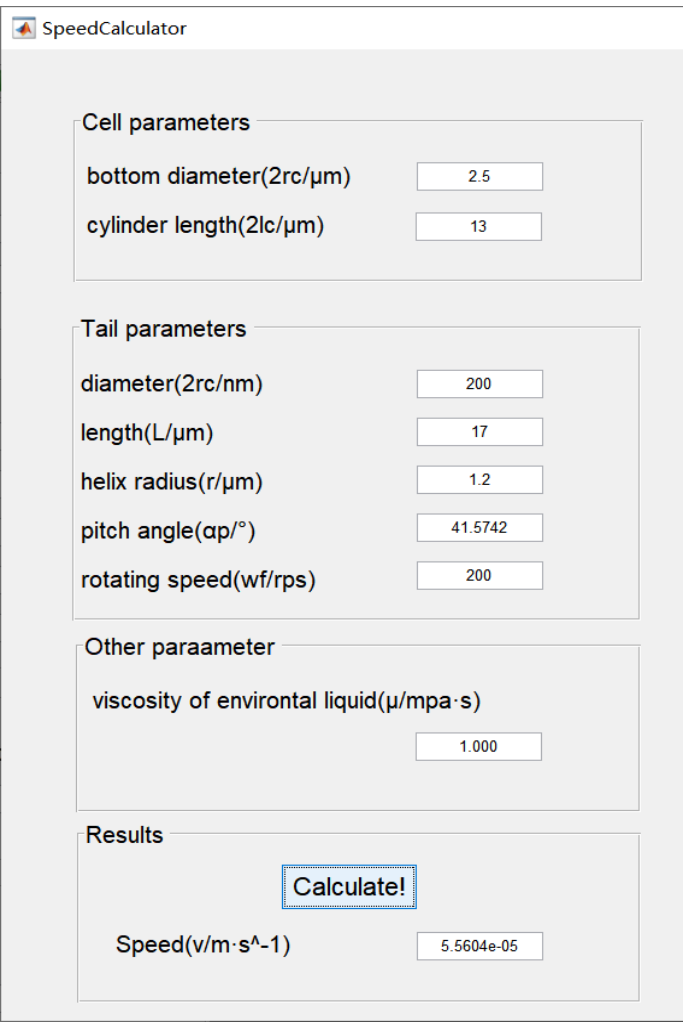

rps means rounds per second, also known as $\mathrm{Hz}$.

Figure 3. Calculation for the original model.

Then, we will focus on the pitch angle and do some optimization work in next part.

\subsection{Optimization Work}

In order to have a good test result in the latter experiments, we hope to use the value near the optimal value when we design the model. If we use such values, the model is able to move as fast as possible. On the one hand, moving faster means the model will have a longer movement track which is easier to measure. On the other hand, a model with a faster speed will be less affected by various kinds of errors arisen from measurement approximation, disturbance from slight water flow or something else.

Now we have determined all parameters as Figure 3 shows. An examination will be done to the value of the pitch angle, since it is a derived quantity. We will fix other parameters' values and change the pitch angle range from $0^{\circ}$ to $90^{\circ}$ and then draw a curve in a rectangular coordinate system taking the pitch angle and the speed as variables of two axes (Pitch angle for $\mathrm{X}$ axe and Speed for $\mathrm{Y}$ axe). After that, we can obtain the optimal value of the pitch angle via searching for the point with the biggest value of the speed.

Another Matlab program with GUI controls based on traditional RFT is applied to do such optimization work. The main code of the program will be presented in Appendix 2. The results are shown in Figure 4 and Figure 5. 


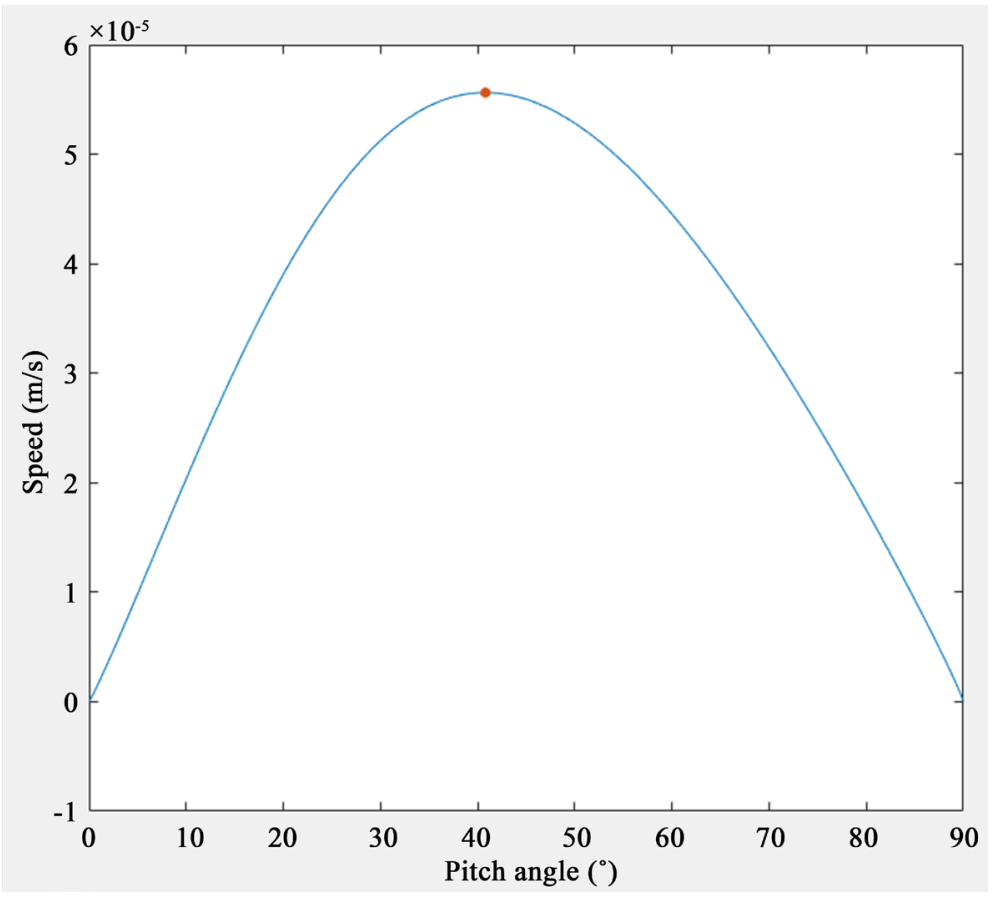

Figure 4. Plot curve.

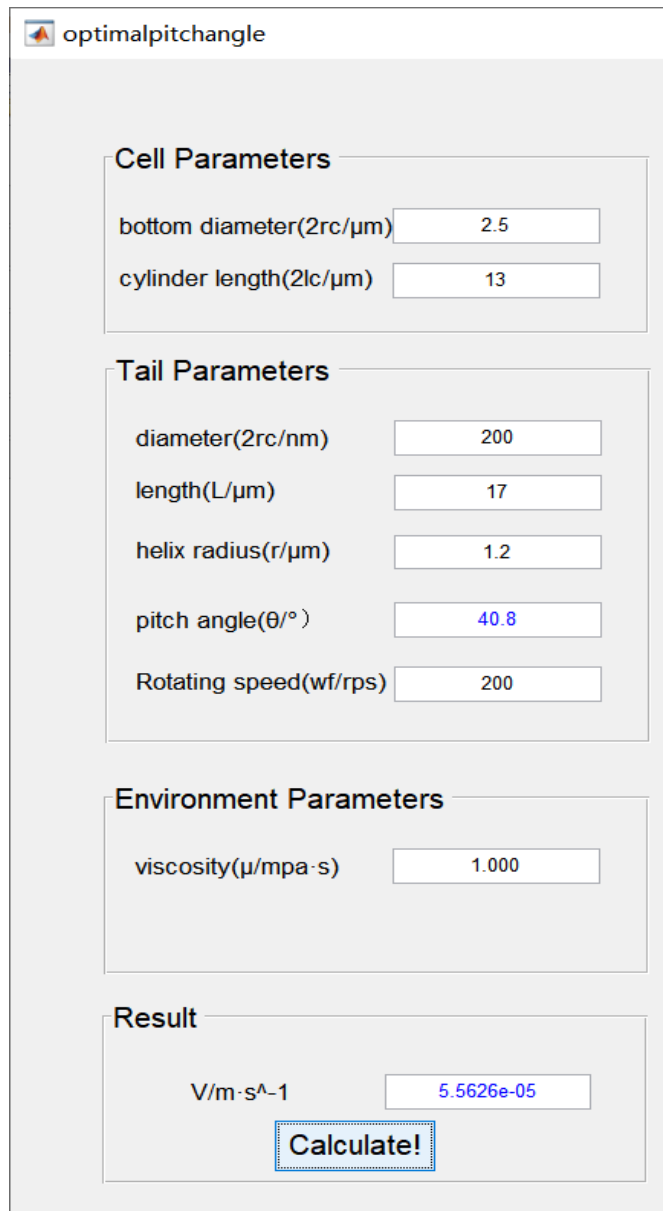

Figure 5. Optimal values of the pitch angle and speed. 
Based on theoretical derivation, we discover that the optimal value of the pitch angel of a flagellum motor should be $45^{\circ}$ or a little bit smaller than $45^{\circ}$ [10] [11] [12]. The result shown above is consistent with the theoretical derivation, which means our optimization work is reliable.

In 3.1, the pitch angle of the original model is shown. The value of $43.3038^{\circ}$ is near the optimal value and it can also be seen that the theoretical value of the speed is quite close to the maximum. AS a result, all parameters' values are determined reasonably.

\section{Scaled Model Building}

\subsection{Model Structure Design}

Figure 6 shows the overall appearance of the real model.

The real model can be generally divided into three parts: the cell body, the connection and the flagellum tail.

The cell body is consisting of two hollow half cylinders, which is shown in Figure 7 as $3 \mathrm{D}$ model parts.

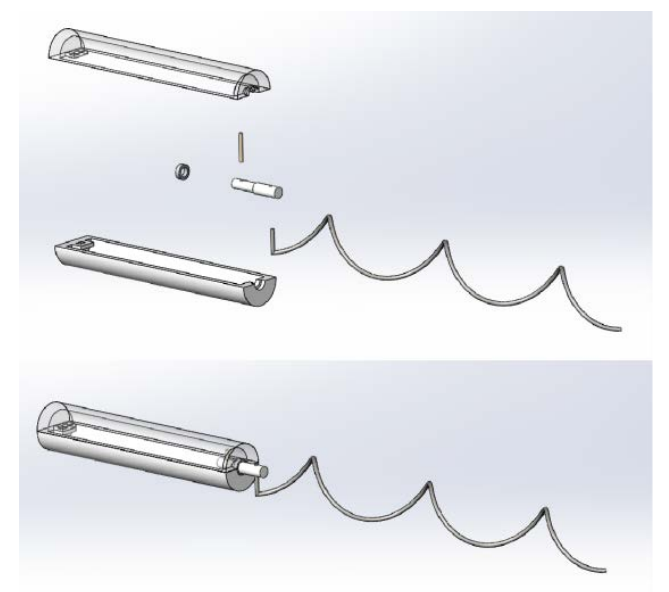

Figure 6. Overall appearance of real model.
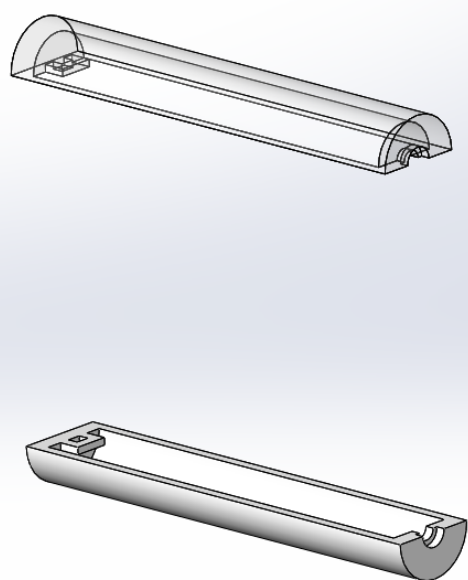

Figure 7. The cell body part. 
They are fixed together with the thin waterproof tape. At the top of the cylinder, a ring is designed to hold rubber bands.

The connection part is composed by the rubber bands, a rotating shaft with a pin and a bearing (Shown in Figure 8 as 3D parts).

Since the real model is an inorganic structure, its tail is not able to rotate spontaneously without any driving link. So, we use the rubber band to provide a torque for the tail to rotate, propelling the whole model to move forward.

Besides, the rotating shaft and the pin are not designed as one part because of consideration of assembling.

The Flagellum tail is an independent part which is shown in Figure 9.

\subsection{Parameters Determination and Material Selection}

Two different scaling ratios (1:500 and 1:1000) are chosen to build the real model.

By testing them in a long water tank, it is found that the 1:500 model cannot float and swim in the water well because the cell body is too small, which cannot provide enough buoyant force for the whole model to swim stably in the water. Additionally, since the cell body cylinder is short, it is hard for a rubber band to work stably for a relative long time. By contrast, the 1:1000 model performs well. This scaled real model can swim a long track and keep swimming stably in a period of time.

We choose to use a PLA 3D printer to manufacture the cell body and the rotating shaft parts. PLA is a kind of light material which contributes to the model's floating in the water. Also, 3D printing can greatly fit complex part models.

Then, some tests are taken to determine the material of the flagellum tail. Tails made of PLA, ferroalloy and steel wire are tested. We mainly focus on the weight and deformation of a tail. There is always a great deformation of tails made of

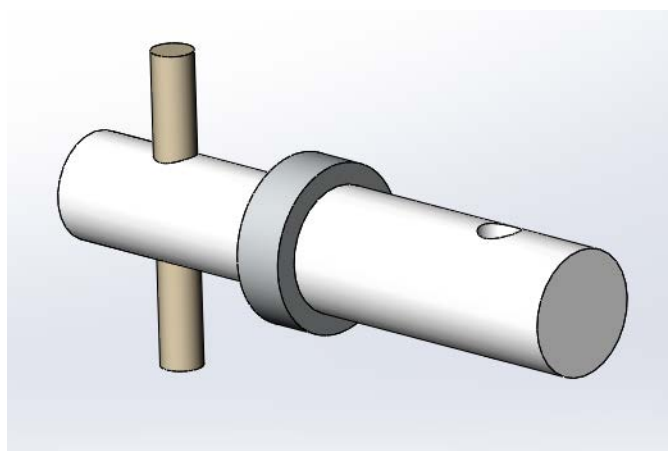

Figure 8. The connection part.

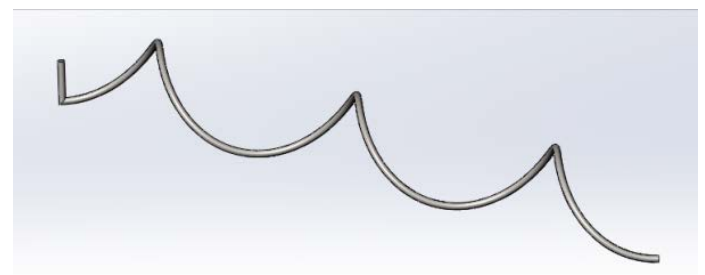

Figure 9. The tail. 
PLA after each test, though they are light enough to float in the water. Figure 10 shows a tail made of PLA with great deformation after several tests.

Tails made of steel wire are too heavy, which makes the head of the cell body rise out of the water. Tails made of ferroalloy wire do not have obvious shortcomings. A little deformation also happens to them, but that will not make them greatly out of shape and affect the movement of the whole model. Finally, we select a tail made of iron wire as the formal model parts.

With all things determined, the real model is built as Figure 11 shows.

\section{Experiment}

\subsection{Scheme}

\subsubsection{Swimming Environment}

We plan to have the real scaled model swim in a long tank ( $1 \mathrm{~m}$ long) with water as Figure 12 shows.

Then, to ensure that traditional RFT and the Pi Theorem can work, we need to create a same Re number environment for the real scaled model. We can replace water with other kinds of liquid with higher value of viscosity.

However, when the viscosity changes, drag coefficients and characteristic velocity of the fluid field will also change. It is quite hard to match the exact Re number. Furthermore, the Re number of the original model's fluid field is so small that it is too hard to get close to it. So, we plan to do several test groups to

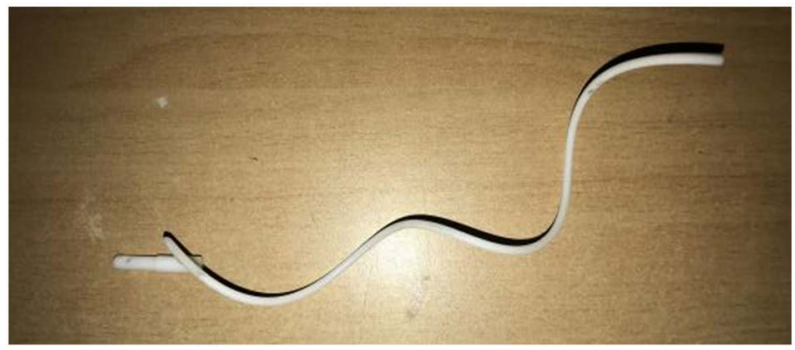

Figure 10. Tail made of PLA.

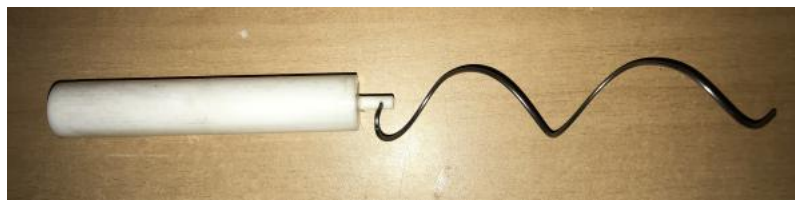

Figure 11. The real model.

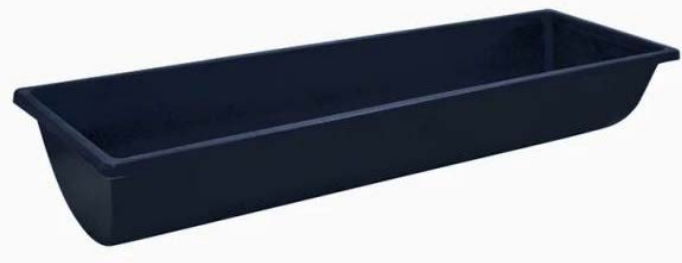

Figure 12. The tank. 
estimate the speed of the real model swimming in the same Re number environment as the original model does.

Tests are planned to be done in the water and a kind of soybean oil. A low Re number environment can be created with such soybean oil with high viscosity. A paper is referred to determine the viscosity value (50 mpa.s) [13] [14]. Meanwhile, a test to evaluate viscosity of the oil is done to examine whether the value offered by the paper is reasonable.

\subsubsection{Viscosity Examination}

A long pipe $(71 \mathrm{~cm})$ full of such oil is used to do this test as Figure 13 shows.

A small metal nut will be released to drop down to the pipe bottom. At the beginning of dropping, the gravity acting on the nut is larger than resultant force of buoyancy and viscous resistance, accelerating the speed of the nut. With the acceleration of the speed, the viscous resistance will increase until the resultant force of buoyancy and viscous resistance is as big as the gravity acting on the nut as the following formula (9) shows. Since then, the nut will drop with a stable speed.

$$
G=F_{b}+f
$$

$G$ refers to the gravity acting on the nut. $\left(G=m g \approx 2 \times 10^{-3} \mathrm{~N}\right) . F_{b}$ is buoyancy and $f$ is the viscous resistance.

The buoyancy can be calculated with Formula (10).

$$
F_{b}=\rho V g
$$

Here, $\rho$ is the density of the soybean oil $\left(920 \mathrm{~kg} / \mathrm{m}^{3}\right)$ and $V$ is the volume of the nut $\left(250 \mathrm{~mm}^{3}\right)$.

The nut can be viewed as a small sphere and then Stokes viscosity formula can be applied to calculate the viscous resistant force acting on the nut.

$$
f=6 \pi \eta r v
$$

Here, $f$ is the viscous resistance and $\eta$ is the viscosity of the liquid. $r$ is the sphere radius $(5 \mathrm{~mm})$ and $v$ is the dropping speed of the nut. The dropping test is recorded as a video for analysis and we find that the dropping time is about $2.7 \mathrm{~s}$ and the dropping speed is about $0.26 \mathrm{~m} / \mathrm{s}$.

Therefore, with Formula (9)-(11) and all these parameters' values, we can calculate the oil's viscosity. The result is about $72.3 \mathrm{mpa} \cdot \mathrm{s}$, which is close to the value mentioned in the paper. So, we will use the value, $72.3 \mathrm{mpa} \cdot \mathrm{s}$, in following calculation.

\subsubsection{Method of Measurement}

Before we test the real model's speed of swimming in the water, we first need to

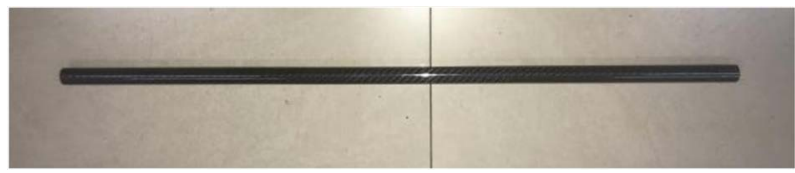

Figure 13. The pipe. 
measure the rotating speed of the flagellum tail. The strategy to obtain the rotating speed of the flagellum tail is to take videos of the model's swimming process. Then, those videos are processed and analyzed frame by frame. A point will be marked on the shaft and we will count the number of the tail's rounds according to the mark during the whole movement. With the number of rounds $\left(N_{r}\right)$ and the time of the movement $(t)$, we can calculate the rotating speed $\left(\omega_{f}\right)$ of the tail with Equation (12).

$$
\omega_{f}=\frac{N_{r}}{t}
$$

Adobe Premiere Pro (PR) CC 2019 is applied to analyze the videos, so the accuracy of time is $0.1 \mathrm{~s}$ in the measurement work.

The speed measurement is also based on the videos. The actual track of the real model's movement is a complex curve. It is impossible for us to obtain the accurate value of the length of the track. Therefore, we need some approximation calculation. We plan to view the track as a section of a circular arc and build a mathematical model as Figure 14 and Figure 15 show. Symbols $I_{t}$ and $w_{t}$ represent the horizontal and vertical offsets respectively. Symbol $\alpha$ is the angle offset of the whole model. Since each time the model will be released paralleling to the long edge of the tank, we can measure the angle between the tank edge and the final speed direction as $\alpha$.

With Alternate Segment Theorem (Equation (13)), Pythagorean Theorem and triangular relation (Equation (14), (15)), we can easily solve the length of the section of circular arc (Equation (16)).

$$
\begin{gathered}
\beta=\frac{\theta}{2}=\alpha-\gamma \\
r \cdot \sin \beta=\frac{\sqrt{l_{t}^{2}+w_{t}^{2}}}{2} \\
\tan \gamma=\frac{w_{t}}{l_{t}} \\
L=r \theta=\frac{l_{t}^{2}+w_{t}^{2}}{l_{t} \sin \alpha-w_{t} \cos \alpha}\left(\alpha-\arctan \left(\frac{w_{t}}{l_{t}}\right)\right)
\end{gathered}
$$

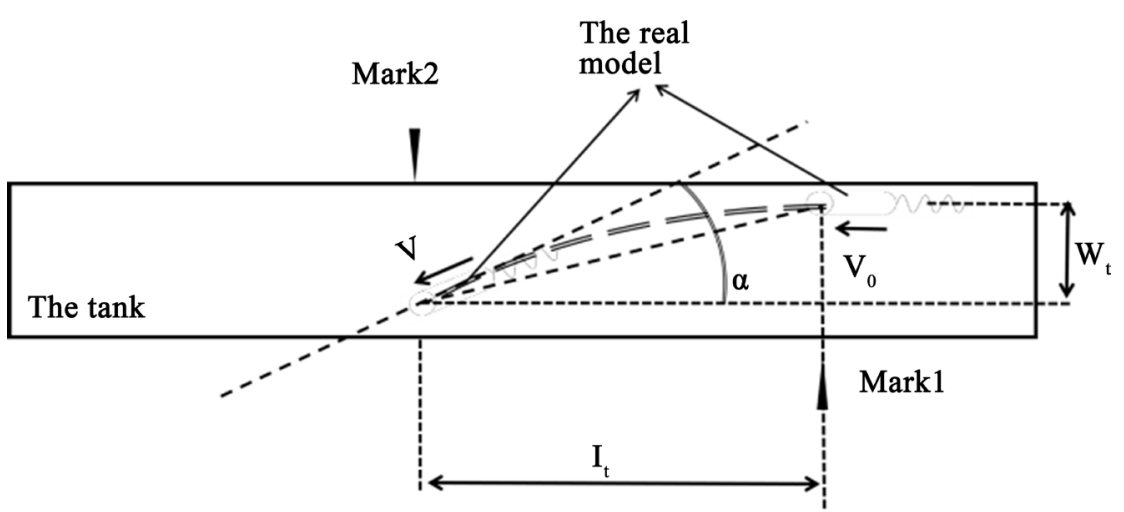

Figure 14. The track model. 


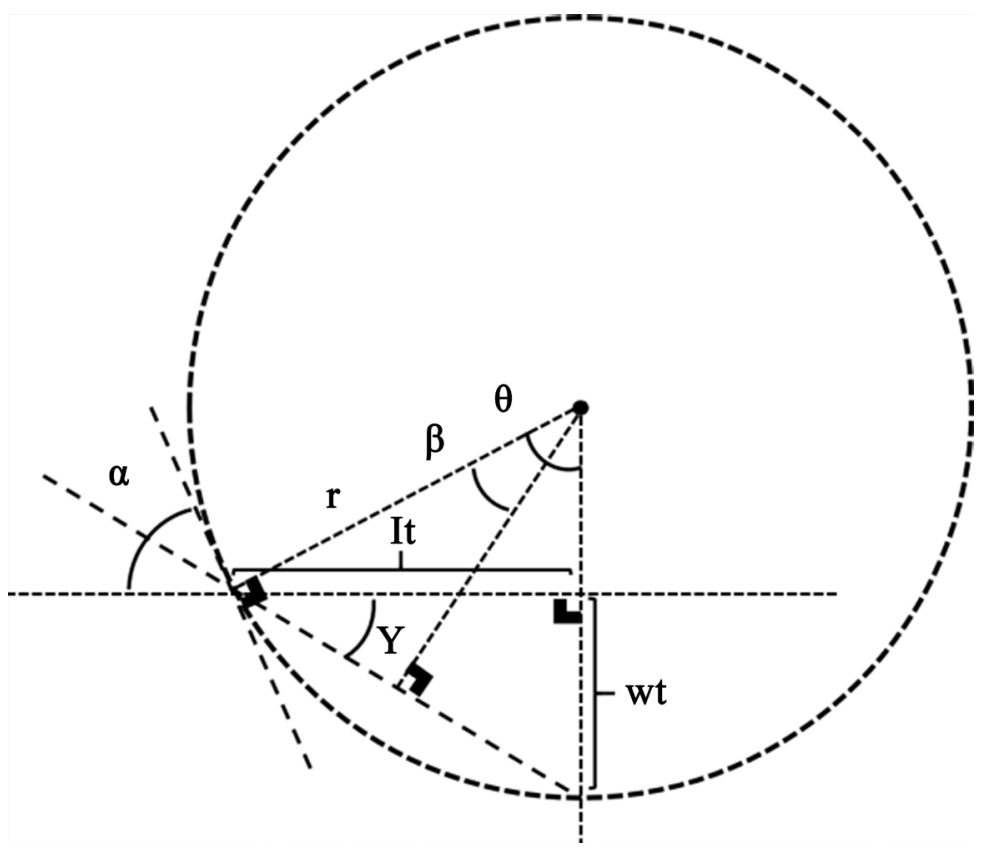

Figure 15. Mathematical model.

Parameters including $l_{t}$ and $w_{t}$ are directly measured during the tests based on the marks. These two parameters are measured by the band tape, so their statistical accuracy is $0.1 \mathrm{~cm}$. Then, we analyze the videos frame by frame to confirm the exact moving time and $\alpha$. Pr CC 2019 and a protractor app are used to do such measurement work, so the statistical accuracy of moving time and $\alpha$ are 0.1 $s$ and $0.1^{\circ}$ respectively.

We will carry out multiple tests for the measurement for both the tail's rotating speed and the speed and calculate the average value as the result. Additionally, among all tests, we will rule out some specific cases which are influenced by accidental outer disturbance.

All test videos are available in.

\subsection{Statistics}

Table 5 lists the all statistics recorded during the tests in water. With Equation (9), we can calculate values of tail's rotating speed in all ten tests. An average value of $9.13 \mathrm{~Hz}$ is obtained. Then, we apply formula 8 to calculate the theoretical value of the scaled flagellum motor's speed. (Here we use the bottom radius of the cell body to represent the parameter Ls which represents model dimensions, because all dimensional parameters are all scaled in the same ratio).

$$
V_{S}=\frac{\omega_{S} L_{S}}{\omega_{O} L_{O}} V_{O}=\frac{9.13 \times 25 \times 10^{-3}}{200 \times 2.5 \times 10^{-6}} \times 4.56 \times 10^{-5}=20.8 \mathrm{~mm} \cdot \mathrm{s}^{-1}
$$

The average speed of the model swimming in water is $241.3 \mathrm{~mm} / \mathrm{s}$. And Re number is about $5 \times 10^{3}$ here. It is much higher than that of original model.

Table 6 lists the all statistics recorded during the tests in the soybean oil. Calculated as those tests done in water, an average value of $6.90 \mathrm{~Hz}$ is obtained. 
Table 5. Statistics of model swimming in water

\begin{tabular}{|c|c|c|c|c|c|c|c|c|}
\hline No. & $\begin{array}{c}\text { Time } \\
(s)\end{array}$ & $\begin{array}{c}\text { Total } \\
\text { Rounds } \\
\text { (r) }\end{array}$ & $\begin{array}{c}a \\
\left({ }^{\circ}\right)\end{array}$ & $\begin{array}{c}I_{t} \\
(\mathrm{~cm})\end{array}$ & $\begin{array}{c}W_{t} \\
(\mathrm{~cm})\end{array}$ & $\begin{array}{c}\text { Track } \\
\text { length } \\
(\mathrm{cm})\end{array}$ & $\begin{array}{c}\text { Rotating } \\
\text { Speed }(\mathrm{rad} / \mathrm{s})\end{array}$ & $\begin{array}{l}\text { Speed } \\
(\mathrm{mm} / \mathrm{s})\end{array}$ \\
\hline 1 & 1.7 & 14 & 23.2 & 34.8 & 20 & 38.10 & 8.24 & 224.1 \\
\hline 2 & 1.8 & 16 & 26 & 50.2 & 20 & 54.09 & 8.89 & 300.5 \\
\hline 3 & 2.7 & 25 & 8.3 & 61.5 & 16.3 & 63.66 & 9.26 & 235.8 \\
\hline 4 & 3.1 & 27 & 22.4 & 61.5 & 15.8 & 63.70 & 8.71 & 205.5 \\
\hline 5 & 2.2 & 20.5 & 6.7 & 58.7 & 14.2 & 60.42 & 9.32 & 274.6 \\
\hline 6 & 2.1 & 18.5 & 6.5 & 49.2 & 15.3 & 51.60 & 8.81 & 245.7 \\
\hline 7 & 2 & 18.5 & 5.3 & 54.5 & 13.5 & 56.15 & 9.25 & 280.8 \\
\hline 8 & 2.9 & 29 & 16.0 & 61.5 & 20 & 64.88 & 10.00 & 223.7 \\
\hline 9 & 3.1 & 28 & 2.5 & 61.5 & 9.5 & 62.27 & 9.03 & 200.9 \\
\hline 10 & 2.8 & 27.5 & 0.0 & 61.5 & 7.6 & 62.01 & 9.82 & 221.4 \\
\hline \multicolumn{7}{|c|}{ average } & 9.13 & 241.3 \\
\hline \multicolumn{7}{|c|}{ standard deviation } & 0.49 & 31.8 \\
\hline
\end{tabular}

The maximum for values of $l_{t}$ and $w_{t}$ are $61.5 \mathrm{~cm}$ and $20 \mathrm{~cm}$ respectively. They are measured before tests and they are determined by the dimensions of the tank and model.

Table 6. Statistics of model swimming in solution

\begin{tabular}{|c|c|c|c|c|c|c|c|c|}
\hline No. & Time (s) & $\begin{array}{c}\text { Total } \\
\text { Rounds } \\
\text { (r) }\end{array}$ & $\begin{array}{c}a \\
\left({ }^{\circ}\right)\end{array}$ & $\begin{array}{c}I_{t} \\
(\mathrm{~cm})\end{array}$ & $\begin{array}{c}W_{t} \\
(\mathrm{~cm})\end{array}$ & $\begin{array}{c}\text { Track } \\
\text { length } \\
(\mathrm{cm})\end{array}$ & $\begin{array}{c}\text { Rotating } \\
\text { Speed } \\
(\mathrm{rad} / \mathrm{s})\end{array}$ & $\begin{array}{c}\text { Speed } \\
(\mathrm{mm} / \mathrm{s})\end{array}$ \\
\hline 1 & 2.2 & 16 & 27.3 & 14.0 & 9.2 & 16.8 & 7.27 & 76.3 \\
\hline 2 & 2.5 & 19 & 26.3 & 15.2 & 12.0 & 19.5 & 7.60 & 78.0 \\
\hline 3 & 2 & 13 & 30.0 & 9.4 & 14.2 & 17.7 & 6.50 & 88.3 \\
\hline 4 & 2.5 & 16.5 & 29.1 & 20.6 & 14.0 & 24.9 & 6.60 & 99.8 \\
\hline 5 & 2.3 & 17 & 23.3 & 18.9 & 12.3 & 22.7 & 7.39 & 98.5 \\
\hline 6 & 2.3 & 16 & 22.8 & 21.1 & 10.5 & 23.6 & 6.96 & 102.5 \\
\hline 7 & 3.1 & 20 & 27.9 & 20.3 & 12.7 & 24.0 & 6.45 & 77.3 \\
\hline 8 & 2.5 & 16 & 27.7 & 19.6 & 12.5 & 23.3 & 6.40 & 93.1 \\
\hline \multicolumn{7}{|c|}{ average } & 6.90 & 89.2 \\
\hline \multicolumn{7}{|c|}{ standard deviation } & 0.47 & 10.8 \\
\hline
\end{tabular}

Then, we apply formula (8).

$$
V_{S}=\frac{\omega_{S} L_{S}}{\omega_{O} L_{O}} V_{O}=\frac{6.90 \times 25 \times 10^{-3}}{200 \times 2.5 \times 10^{-6}} \times 4.56 \times 10^{-5}=15.7 \mathrm{~mm} \cdot \mathrm{s}^{-1}
$$

The average speed of the model swimming in the soybean oil is $8.9 \mathrm{~mm} / \mathrm{s}$. Here, Re number is about $3 \times 10^{1}$. 


\section{Discussion}

\subsection{Value Analysis}

Firstly, as we have mentioned in 2.1, we apply the approximation calculation when we calculate the drag coefficient of the cell body. The real model is shaped like a cylinder instead of $\mathrm{n}$ a spheroid, this will cause a greater viscosity resistance force, which slows the model's movement. This error could be eliminated by modifying the shape of the cell body model part.

Secondly, the real model will have some manufacturing and assembly errors. Manufacturing errors will cause any possible errors, while assembly errors will lead to redundant actions and frictions between which cause extra energy loss during the movement.

Thirdly, we apply approximation during the measurement work as well. This will also cause some errors. Furthermore, during the process of measuring, we have measuring errors as well.

Taking all these errors into consideration, the speed value tested in the experiment should be slower than that tested in an ideal situation.

\subsection{Verification}

We hope to verify the application feasibility of Buckingham Pi Theorem in such design of flagellum motor, but the result statistics do not accord with our expectation. That is due to the fact that we are not able to create a low Re number environment for the real model.

However, we can make some inferences from the test statistics.

Comparing the two test groups, we can find that when we test the model in an environment with a lower Re number, the final result value of the model's speed is lower. Surely, since the rotating speed of the tail is slower as well, the theoretical value of the model's speed will decrease. But, obviously, the experimental value decreases much faster than the theoretical value. We can infer that if we replace the environmental liquid with greater viscosity which contributes lower Re number until it is near the Value $3 \times 10^{-4}$, we would finally find that the experimental value is smaller than the theoretical value. And combined with analysis shown in 6.1, we can make a primary conclusion that Buckingham Pi Theorem can be applied to such designs of a scaled flagellum motor model.

\subsection{Experiment Improvement}

A lot of work to improve the experiment has been discussed in 6.1 , so here we only include some other aspects.

Firstly, since we only make two sets of experiments and can only see an inclination that the theoretical value of the speed would be smaller than the experimental results. To confirm it more exactly, we should keep trying to increase the viscosity of the environment liquid and do more tests until the Re number gets close to the Re number of the original model. Then, we find out whether the experimental results are just a little bit smaller than the theoretical value. This 
process will make our verification work more logical and rigorous.

Secondly, the building of the real scaled model can be optimized by replacing some parts. For example, we can try to replace the rubber band with a small motor. During the process of the real model's movement, the torque provided by the rubber band decays over time, which make the model speed and tail's rotation speed uneven. A motor could offer the model a stable torque and we can focus on the middle part of the whole movement when the real model moves at a stable speed and its tail rotates uniformly. This way we are able to obtain more reliable statistics from tests.

Thirdly, we can choose a more suitable environment for the real model to swim. A larger area allows the real model to move as far as possible and its moving will not be interrupted by any margins or boundaries. This way, we can promise that the model's movement would be affected by less interference.

\subsection{Future}

As a kind of practical and efficient structure, the flagellum motor will be applied to more fields if its suitable dimensions can be precisely calculated out. Maybe one day we can find some big machines adapted from flagellum motor structure work for sailing in silt; and it may come true that flagellum motor structure is widely used as the propeller of micro-robots applied in medical fields. Along with the development of material science and manufacturing technologies, we will have more innovative related designs of all sizes. At that time, Buckingham Pi Theorem will be more meaningful when applied in such design work.

\section{Conflicts of Interest}

The author declares no conflicts of interest regarding the publication of this paper.

\section{References}

[1] Jian, F. and Sung, K.C. (2014) Mini and Micro Propulsion for Medical Swimmers. Micromachines, 5, 97-113. https://doi.org/10.3390/mi5010097

[2] Mohamed, K.T., Ata, A.A. and El-Souhily, B.M. (2011) Dynamic Analysis Algorithm for a Micro-Robot for Surgical Applications. International Journal of $\mathrm{Me}$ chanics and Materials in Design, 7, 17-28. https://doi.org/10.1007/s10999-010-9146-y

[3] Editorial Department (2014) Micro Medical Robot for Targeted Drug Delivery. Shanghai of Biomedical Engineering, No. 2, 118.

[4] Purcell, E.M. (1977) Life at Low Reynolds Number. American Journal of Physics, 45, 3-11. https://doi.org/10.1119/1.10903

[5] Holwill, M.E.J. and Burge, R.E. (1963) A Hydrodynamic Study of the Motility of Flagellated Bacteria. Archives of Biochemistry and Biophysics, 101, 249-260. https://doi.org/10.1016/S0003-9861(63)80010-7

[6] Buckingham, E. (1914) On Physically Similar Systems; Illustrations of the Use of Dimensional Equations. Physical Review, 4, 345.

https://doi.org/10.1103/PhysRev.4.345 
[7] Magariyama, Y. and Kudo, S. (2002) A Mathematical Explanation of an Increase in Bacterial Swimming Speed with Viscosity in Linear-Polymer Solutions. Biophysical Journal, 83, 733-739. https://doi.org/10.1016/S0006-3495(02)75204-1

[8] Happel, J. and Brenner, H. (1973) Low Reynolds Number Hydrodynamics. Kluwer Academic Publishers, Dordrecht.

[9] Magariyama, Y., Sugiyama, S., Muramoto, K., Kawagishi, I., Imae, Y. and Kudo, S. (1995) Simultaneous Measurement of Bacterial Flagellar Rotation Rate and Swimming Speed. Biophysical Journal, 69, 2154-2162. https://doi.org/10.1016/S0006-3495(95)80089-5

[10] Lighthill, J. (1996) Helical Distributions of Stokeslets. Journal of Engineering Mathematics, 30, 35-78. https://doi.org/10.1007/978-94-009-0225-1_4

[11] Chwang, A.T. and Wu, T.Y.T. (1975) Hydromechanics of Low-Reynolds-Number Flow. Part 2. Singularity Method for Stokes Flows. Journal of Fluid Mechanics, 67, 787-815. https://doi.org/10.1017/S0022112075000614

[12] Lauga, E. and Thomas, R. (2008) The Hydrodynamics of Swimming Microorganisms, Powers, 10.1088/0034-4885/72/9/096601.

[13] Fan, W.L. and Zhang, B.A. (2008) Viscosity Research on Three Kinds of Liquid Detergents. China Surfactant Soap and Detergent Industries, No. 4, 73-74.

[14] Su, H.K. and Zhao, J.T. (2018) Study on the Influence of Temperature on the Viscosity and Density of Vegetable Oil. Cereal \& Food Industry, 25, 29-32. 


\section{Appendix 1}

function pushbutton1_Callback(hObject, eventdata, handles) $\mathrm{rc}=0.0000005^{\star}$ str2double(get(handles.edit 1, 'String' $\left.^{\prime}\right)$ ); $\mathrm{lc}=0.0000005^{\star}$ str2double(get(handles.edit2,'String')); $\mathrm{rf}=0.0000000005^{\star}$ str2double(get(handles.edit3,'String')); $\mathrm{L}=0.000001^{*}$ str2double(get(handles.edit4,'String')); $\mathrm{r}=0.000001^{\star}$ str2double(get(handles.edit5,'String')); $\mathrm{pa}=\operatorname{atan}\left(\left(2^{*} \mathrm{pi}^{*} \mathrm{r}\right) /(\mathrm{L} / 2)\right)$;

$\mathrm{wf}=$ str2double(get(handles.edit7,'String'));

$\mathrm{u}=0.001^{\star}$ str2double(get(handles.edit13,'String'));

$\mathrm{p}=\mathrm{L} / 2$;

set(handles.edit6,'String',num2str(180* pa/pi));

$\% \mathrm{ac} \& \mathrm{bc}$

$\mathrm{ac}=-6^{\star} \mathrm{pi}^{\star} \mathrm{u}^{\star} \mathrm{rc} \mathrm{c}^{\star}\left(1-0.2^{\star}(1-\mathrm{lc} / \mathrm{rc})\right)$;

set(handles.edit8,'String',num2str(ac));

$\mathrm{bc}=-8^{\star} \mathrm{pi}^{\star} \mathrm{u}^{\star} \mathrm{rc}^{\star} \mathrm{rc} \mathrm{r}^{\star} \mathrm{rc}\left(1-0.6^{\star}(1-\mathrm{lc} / \mathrm{rc})\right)$;

set(handles.edit9,'String',num2str(bc));

\%af\&bf\&cf\&K0\&v

$\mathrm{op}=2^{\star} \mathrm{pi}^{\star} \mathrm{u}^{\star} \mathrm{L} /\left(\log 10\left(0.5^{\star} \mathrm{rf} / \mathrm{p}\right)+0.5\right) /\left(4^{*} \mathrm{pi}^{*} \mathrm{pi}^{\star} \mathrm{r}^{\star} \mathrm{r}+\mathrm{p}^{*} \mathrm{p}\right) ;$

af $=o p^{*}\left(8^{*}\right.$ pi $\left.^{*} \mathrm{pi}^{\star} \mathrm{r}^{\star} \mathrm{r}+\mathrm{p}^{*} \mathrm{p}\right)$;

$\mathrm{bf}=\mathrm{op}^{*}\left(2^{*} \mathrm{p}^{*} \mathrm{p}+4^{*} \mathrm{pi}^{*} \mathrm{pi}^{\star} \mathrm{r}^{\star} \mathrm{r}\right)^{*} \mathrm{r}^{*} \mathrm{r}$;

$\mathrm{yf}=\mathrm{op}^{\star}\left(-2^{\star} \mathrm{pi}^{\star} \mathrm{r}^{\star} \mathrm{r}^{\star} \mathrm{p}\right)$;

$\mathrm{K} 0=-\mathrm{wf} /\left(\mathrm{ac}^{*} \mathrm{bc}+\mathrm{af}{ }^{*} \mathrm{bc}\right)$;

$\mathrm{v}=-\mathrm{wf} \mathrm{f}^{*} \mathrm{yf} /(\mathrm{ac}+\mathrm{af})$;

set(handles.edit10,'String',num2str(af));

set(handles.edit11,'String',num2str(bf));

set(handles.edit12,'String',num2str(yf));

set(handles.edit30,'String',num2str(K0));

set(handles.edit31,'String',num2str(v));

\section{Appendix 2}

function pushbutton1_Callback(hObject, eventdata, handles) $\mathrm{rc}=0.0000005^{\star} \operatorname{str} 2$ double (get(handles.edit1,'String')); $\mathrm{l} c=0.0000005^{\star} \operatorname{str} 2$ double (get(handles.edit2,'String')); $\mathrm{rf}=0.0000000005^{\star}$ str2double(get(handles.edit3,'String')); $\mathrm{L}=0.000001^{\star}$ str2double(get(handles.edit4,'String')); $\mathrm{r}=0.000001^{\star}$ str2double(get(handles.edit5,'String')); $\mathrm{pa}=0: 0.1: 90$;

$\mathrm{wf}=\mathrm{str} 2 \mathrm{double}$ (get(handles.edit7,'String'));

$\mathrm{u}=0.001^{\star}$ str2double(get(handles.edit8,'String')); 


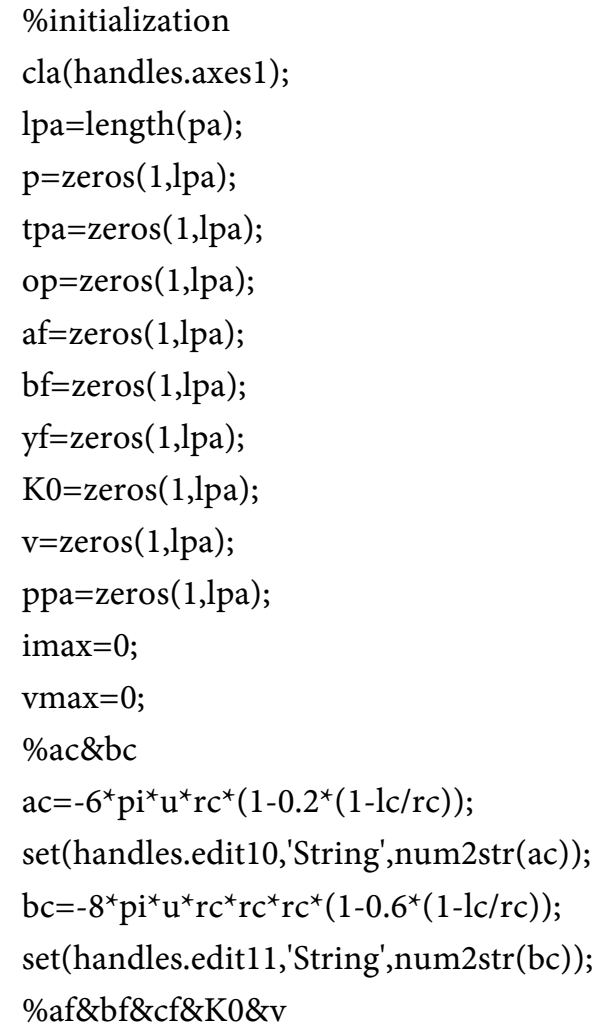


O. L. Jin

set(handles.edit14,'String',num2str(yf(imax)));

set(handles.edit15,'String',num2str(K0(imax)));

set(handles.edit16,'String',num2str(v(imax)),'Foregroundcolor','Blue'); 